\title{
Simulation of rapid startup in microwave magnetrons with azimuthally varying axial magnetic fields
}

\author{
M. C. Jones, V. B. Neculaes, W. White, Y. Y. Lau, ${ }^{\text {a) }}$ and R. M. Gilgenbach \\ Intense Energy Beam Interaction Laboratory, Department of Nuclear Engineering and Radiological \\ Sciences, University of Michigan, Ann Arbor, Michigan 48109-2104
}

(Received 22 August 2003; accepted 10 December 2003)

\begin{abstract}
A method is proposed whereby the startup of a magnetron may be hastened. For a N-cavity magnetron operating in the pi mode, the formation of the N/2 electron spokes is much more rapid when an azimuthally varying axial magnetic field of N/2 periodicity is employed. Electromagnetic particle-in-cell code simulations are presented that show electron prebunching by the azimuthally varying axial magnetic field, long before the pi mode is excited. (C) 2004 American Institute of Physics. [DOI: 10.1063/1.1646225]
\end{abstract}

Some 60 million microwave ovens are sold worldwide on an annual basis. The magnetrons in these ovens typically operate at $1 \mathrm{~kW}$ average power at a frequency of about 2.45 $\mathrm{GHz}$. As this unlicensed part of the microwave frequency spectrum becomes more congested with wireless communication systems, wireless computer local area networks $\left(\right.$ IEEE802.11b and IEEE802.11g) ${ }^{1}$ and Bluetooth, ${ }^{2}$ the issue of interference from oven magnetron microwave noise has become crucial. ${ }^{3}$ Noise in crossed-field amplifiers (CFAs) also significantly limits the signal-to-noise ratio in military radar. Startup of magnetrons in the desired mode has important implications in microwave noise generation. Previously, more rapid magnetron startup has been achieved by microwave priming, an expensive technique applied to high-power (multi-MW) magnetrons. ${ }^{4}$

Several ways are known to hasten the startup of an oscillator or to shorten the linear growth regions of an amplifier. Examples of current interest in the case of linear beam tubes are the emission gated field emitter array ${ }^{5}$ and its photoassisted variants, ${ }^{6}$ where the electrons are bunched at the cathode into the desired mode, before their entrance into the interaction region. In the case of a crossed-field device, in particular, the magnetron, there is no beam to speak of, and the interaction region and the "beam" formation region are one and the same. ${ }^{7}$ This perhaps accounts for the apparent lack of previous attempts of "electron prebunching" on crossed-field devices. Startup of oscillation has to await the mature development of the electron spokes, which need to be built up from noise, and the subsequent excitation of the desired mode. That mode itself needs to be present for the spokes to be formed in the first place. In fact, startup of magnetrons is known to be problematic even in computer simulation. ${ }^{8}$

In a $\mathrm{N}$-cavity magnetron which operates, say, in the $p i$ mode, there are N/2 electron spokes when the oscillation starts. To circumvent the slow startup in such a magnetron, we have tested "prebunching the electrons" by introducing N/2 azimuthal variations in the axial magnetic field. Thus, the number of maxima in the axial magnetic field is N/2 in the azimuthal direction. (The number of minima of the axial magnetic field is also N/2 in the azimuthal direction.) The

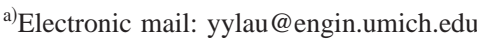

physical reason for this magnetic field arrangement is that when the magnetron is turned on, the electron orbits immediately move into a N/2 fold symmetry which favors the excitation of the pi mode, long before this internal electromagnetic mode appears. These electrons, favorably grouped into N/2 fold symmetry, naturally speed up the excitation of the $p i$ mode in this case.

One might wonder if the azimuthal perturbation in the external magnetic field would reduce the operational efficiency of the magnetron. In recent experiments at the University of Michigan, ${ }^{9}$ we added 4 magnets, placed azimuthally around one of the magnets in a 10 cavity microwave oven magnetron. This magnetron operated in the pi mode. We found the surprising results that the close-in noise and the sideband noise have been completely eliminated, with little sacrifice in the efficiency and output power. In those experiments, we also observed a cleaner microwave spectrum at startup. Note that those previous experiments were not optimized for startup with five azimuthal variations in the external magnetic field.

While the geometrical arrangement in the magnetic fields in the low-noise magnetron experiments ${ }^{9}$ is three dimensional, to demonstrate the feasibility of electron prebunching by imposing an appropriate azimuthal variation in the axial magnetic field, we use a two-dimensional (2D) simulation on the well-studied Bekefi-Palevsky A-6 magnetron. ${ }^{10}$ We use the 2D MAGIC code ${ }^{11}$ and impose an azimuthally varying axial magnetic field of general form (Fig. 1)

$$
B_{z}(\theta)=B_{0}\{1+(\alpha / 2) \sin [n(\theta+p)]\},
$$

where $B_{0}$ is the mean axial magnetic field, $\alpha$ is the magnitude of the maximum azimuthal variation ( $\theta$ variation) of the axial magnetic field (in fractions of the mean magnetic field) in the $n$-fold symmetry, and $p$ is the angular position of the mean magnetic field with respect to the neighboring cavity. In Fig. 1(a), $p=0^{\circ}$; in Fig. 1(b), $p=30^{\circ}$. This function of the magnetic field permits comparisons of electron spoke formation for the baseline case of uniform axial magnetic field $(\alpha=0)$ to axial magnetic field sinusoidal perturbations of different magnitude $(\alpha)$, periodicity $(n)$, and phase $(p)$. The electron beam voltage $(-350 \mathrm{kV})$ of the magnetron used 


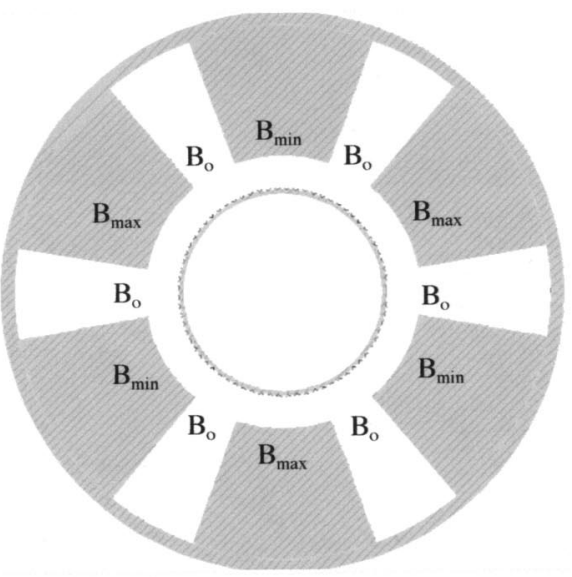

(a)

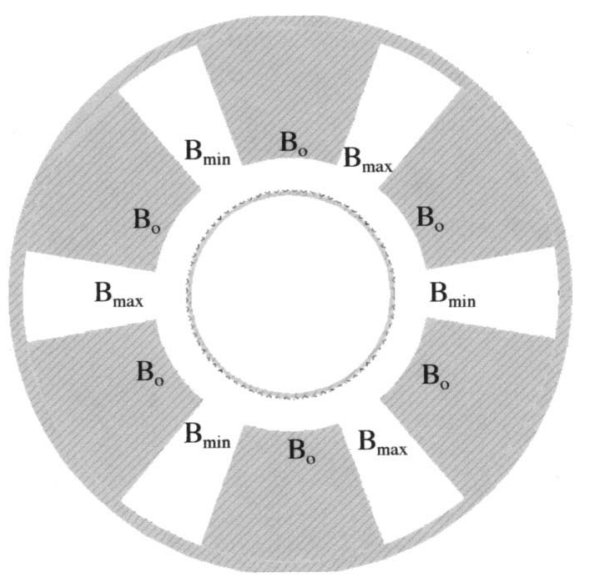

(b)

FIG. 1. Simulation geometries for rapid magnetron startup: (a) mean magnetic field at the cavity $\left(p=0^{\circ}\right)$ and (b) mean magnetic field $30^{\circ}$ from the cavity $\left(p=30^{\circ}\right)$.

in these simulations is similar to that of the high-power magnetron experiments underway at the University of Michigan using the MELBA-C Accelerator. ${ }^{12}$

To encourage rapid excitation of the pi mode, $n=\mathrm{N} / 2$ $=$ threefold symmetry is imposed in the axial magnetic field for the configuration shown in Fig. 1. The parameters in Eq. (1) for this example are $B_{0}=7.2 \mathrm{kG}$ and $n=3$. Figures $2-5$ compare the results of the simulations to an unperturbed (uniform) magnetic field with $\alpha=0$ and a perturbed magnetic field with $\alpha=0.3$. Figures 2 and 3 correspond to the case of $p=0^{\circ}$ [Fig. 1(a)] and Figs. 4 and 5 correspond to the case of $p=30^{\circ}$ [Fig. 1(b)].

Figure 2 shows the electrons in a six cavity magnetron for a very early time in the magnetron pulse (1.186 ns). In the unperturbed magnetic field case [Fig. 2(a)], the electrons in the Brillouin hub show no special feature. In Fig. 2(b), the perturbed magnetic field case, the electrons have clearly begun to form three bunches, the desired number of bunches for $p i$ mode operation in a six cavity magnetron. Note that the formation of these three electron bunches is due solely to the threefold azimuthal symmetry in the external axial magnetic field, long before the pi mode is excited.

Figure 3 illustrates the electron positions for the magnetron at a time (5.368 ns) when bunching has begun in the unperturbed magnetic field case [Fig. 3(a)]. In the perturbed magnetic field case [Fig. 3(b)], the electron spokes are fully

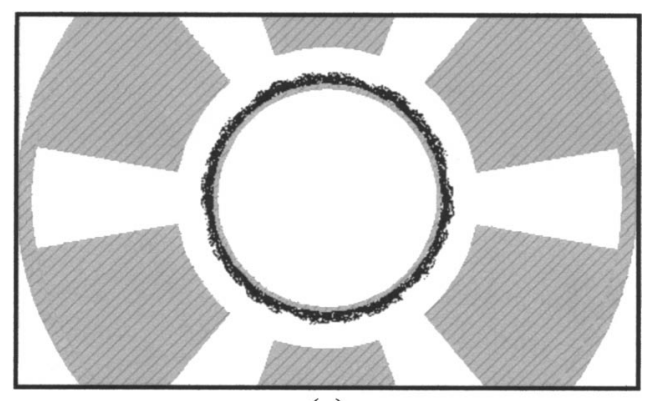

(a)

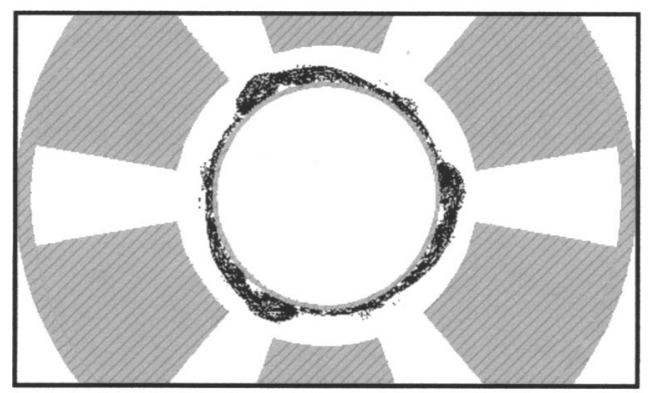

(b)

FIG. 2. Electron positions in the magnetron simulation at the early time, 1.186 ns: (a) unperturbed axial magnetic field and (b) perturbed axial magnetic field with $\alpha=0.3, n=3$, and $p=0^{\circ}$.

developed and extend well into the magnetron cavities; it is expected that microwave oscillation would begin to develop at this time.

Rapid prebunching of electrons at early times has also been observed for other values of $p$. Figure 4 depicts the electron positions in the magnetron for the case of $p=30^{\circ}$ [Fig. 1(b)]. It is taken at $5.269 \mathrm{~ns}$, about the same time as Fig. 3, which is for the case of $p=0^{\circ}$ [Fig. 1(a)]. Again, for the unperturbed magnetic field case, Fig. 4(a) shows only the

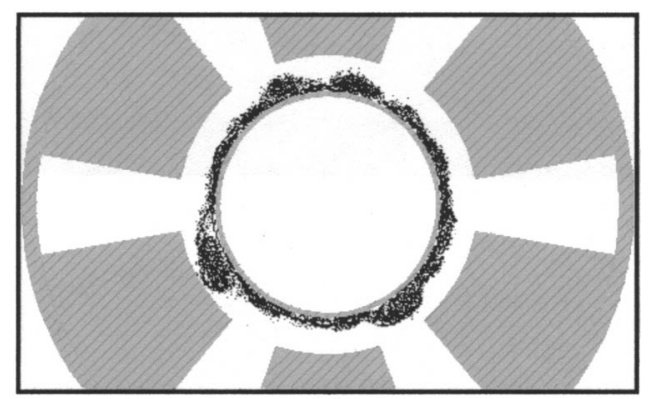

(a)

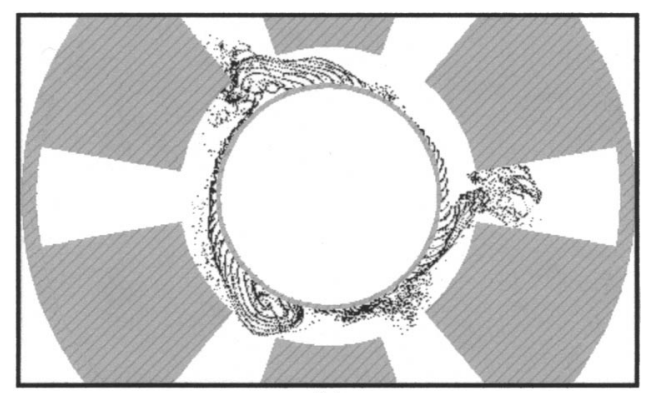

(b)

FIG. 3. Electron positions in the magnetron simulation at $5.368 \mathrm{~ns}$ : (a) unperturbed axial magnetic field and (b) perturbed axial magnetic field with $\alpha=0.3, n=3$, and $p=0^{\circ}$. 


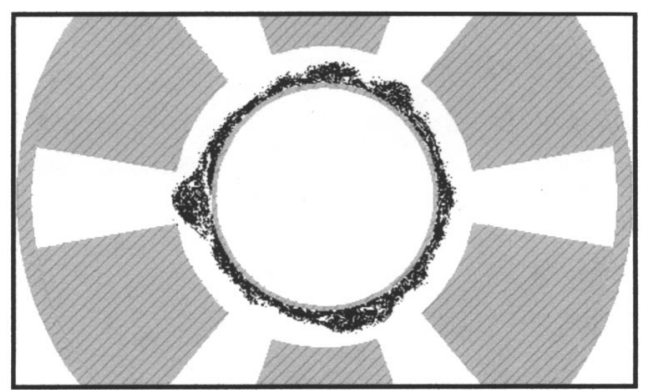

(a)

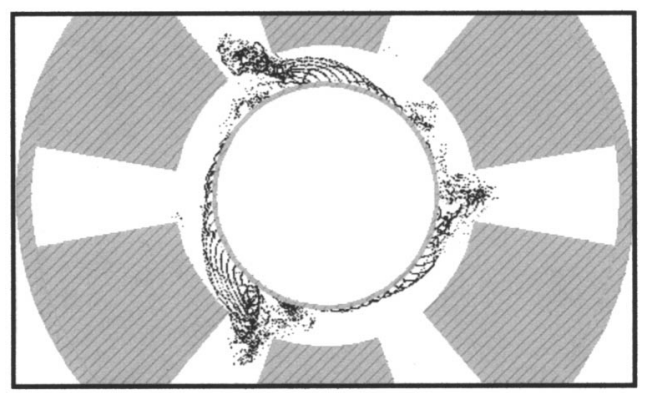

(b)

FIG. 4. Electron positions in the magnetron simulation at 5.269 ns: (a) unperturbed axial magnetic field and (b) perturbed axial magnetic field with $\alpha=0.3, n=3$, and $p=30^{\circ}$.

beginning of electron bunching. In contrast, for the perturbed magnetic field case [Fig. 4(b)], the three electron spokes are fully developed and extend well into the magnetron cavities and the pi mode is expected to begin to be excited by this time. Figure 5 is the same as Fig. 4, but is taken at a still later time, $9.945 \mathrm{~ns}$, when the three spokes in the unperturbed magnetic field case are close to their mature state [Fig. 5(a)]. For the perturbed magnetic field case [Fig. 5(b)], the spokes are already well developed, since they retain the same feature

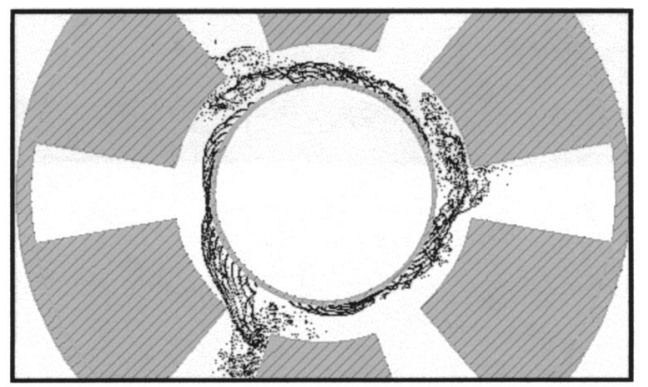

(a)

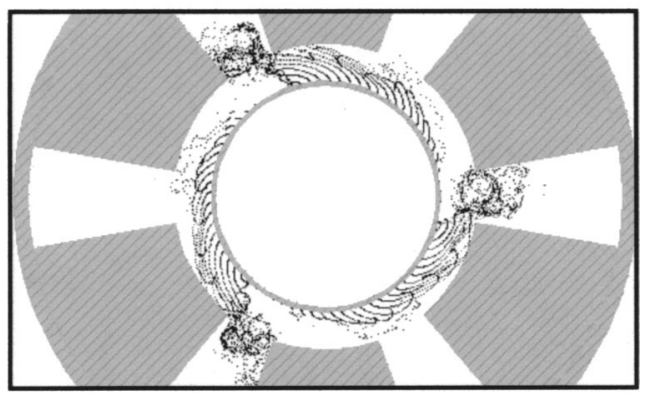

(b)

FIG. 5. Electron positions in the magnetron simulation at 9.945 ns: (a) unperturbed axial magnetic field and (b) perturbed axial magnetic field with $\alpha=0.3, n=3$, and $p=30^{\circ}$. as at the earlier time, 5.269 ns, as shown in Fig. 4(b). Thus, the perturbed magnetic field case hastens the startup by about a factor of 2 in time in this example. These simulations suggest that rapid startup is achieved for both phases shown in Figs. 1(a) and 1(b).

Rapid spoke formation is anticipated even from single particle orbit considerations, since there is almostinstantaneous kinematic bunching of the electron cycloidal orbits when there is azimuthal variation in the axial magnetic field. In the present simulation, we have monitored the total (radial) current that passes through a cavity gap. Mature variation of this current, corresponding to a spoke entering the specified cavity, is observed around $5 \mathrm{~ns}$ for the perturbed magnetic field $\left(p=0^{\circ}\right)$ and around 9 ns for the unperturbed magnetic field.

In summary, the simulations demonstrate that azimuthally varying axial magnetic fields cause more rapid formation of the electron spokes in magnetrons. Furthermore, in a $\mathrm{N}$ cavity magnetron, an azimuthally varying magnetic field of N/2 periodicity causes rapid formation of the N/2 spokes characteristic of the pi mode. Our most recent experiments, modified from those in Ref. 9, do confirm that fivefold azimuthal magnetic field symmetry in the 10 cavity magnetron produces low-noise operation in the pi mode, beginning at the lowest anode current. These experimental results will be reported elsewhere. This technique may be considered a crossed-field analog of electron prebunching, and could prove useful in both oven magnetrons and high power relativistic magnetron startup and mode control. It could also be adopted to shorten the interaction length of crossed-field amplifiers.

This research was supported by the DUST (S\&T) under the Innovative Microwave Vacuum Electronics MURI Program managed by the U.S. Air Force Office of Scientific Research under Grant No. F49620-99-1-0297. Sponsorship was also received from the Air Force Office of Scientific Research under Grant Nos. F49620-00-1-0088 and F4962002-1-0089. The authors appreciate AFOSR support of the MAGIC Users Group provided by Mission Research Corporation.

${ }^{1}$ See, for example, http://grouper.ieee.org/groups/802/11/.

${ }^{2}$ See, for example, http://www.bluetooth.com/.

${ }^{3}$ J. M. Osepchuk, IEEE Trans. Microwave Theory Tech. 50, 975 (2002).

${ }^{4}$ J. Robinson, M. Doherty, B. Davenport, M. Lander, and T. Treado, Proceedings of the 3rd IEEE International Vacuum Electronics Conference, Monterey, CA, 23-25 April (IEEE Piscataway, NJ, 2002), p. 24.

${ }^{5}$ D. R. Whaley, B. M. Gannon, V. O. Heinen, K. E. Kreischer, C. E. Holland, and C. A. Spindt, IEEE Trans. Plasma Sci. 30, 998 (2002).

${ }^{6}$ K. L. Jensen, Y. Y. Lau, and D. McGregor, Appl. Phys. Lett. 77, 585 (2000); Solid-State Electron. 45, 831 (2001).

${ }^{7}$ See, for example, Y. Y. Lau, in High Power Microwave Sources, edited by V. L. Granatstein and I. Alexeff (Artech House, Norwood, MA, 1987), Chap. 9.

${ }^{8} \mathrm{~J}$. W. Luginsland, private communications.

${ }^{9}$ V. B. Neculaes, R. M. Gilgenbach, and Y. Y. Lau, Appl. Phys. Lett. 83, 1938 (2003).

${ }^{10}$ A. Palevsky, doctoral dissertation, Massachusetts Institute of Technology, Cambridge, MA, 1980.

${ }^{11}$ B. Goplan, L. Ludeking, D. Smithe, and G. Warren, Comput. Phys. Commun. 87, 54 (1995).

${ }^{12}$ M. R. Lopez, R. M. Gilgenbach, D. W. Jordan, S. A. Anderson, M. D. Johnston, M. W. Keyser, H. Miyake, C. W. Peters, M. C. Jones, V. Bogdan Neculaes, Y. Y. Lau, T. A. Spencer, J. W. Luginsland, M. D. Haworth, R. W. Lemke, and D. Price, IEEE Trans. Plasma Sci. 30, 947 (2002). 